\title{
Infrared Stokes Polarimeter at NAOJ/Mitaka
}

\author{
Yoichiro Hanaoka, Takashi Sakurai, \& IRMag Group \\ National Astronomical Observatory of Japan, \\ 2-21-1 Osawa, Mitaka, Tokyo 181-8588, Japan \\ email: hanaoka@solar.mtk.nao.ac.jp
}

\begin{abstract}
We have been operating an infrared Stokes spectro-polarimeter, whose observing wavelength bands include the He I $10830 \AA$ and Fe I $15648 \AA$ lines. A couple of full-Sun, full-Stokes maps in both wavelength bands are taken on a daily basis, with the polarization sensitivity better than $10^{-3}$. With this sensitivity, the helium polarization maps clearly show the atomic and Hanlé polarizations besides the Zeeman polarization, particularly in prominences/filaments. On these polarization maps, we can track the magnetic field signals of the prominences/filaments during their passages on the solar disk. Therefore, this instrument works as a 'synoptic prominence magnetograph'. Our preliminary study of the linear polarizations seen in quiescent filaments suggests that the magnetic field producing the polarization signals is located at the bottom part of a flux-rope, which supports a filament.
\end{abstract}

Keywords. Polarimetry, Prominences, Magnetic Field

\section{Introduction and the Instrument}

A full-Sun spectro-polarimeter, which aims at studying the evolution of the solar dynamo with a long-term operation, was installed onto the Solar Flare Telescope (Sakurai et al. 1995) at NAOJ/Mitaka (Hanaoka et al., 2011). The observing wavelengths are He I $10830 \AA /$ Si I $10827 \AA$ (for the chromospheric and photospheric magnetic field) and Fe I $15648 \AA(\mathrm{g}=3) / 15653 \AA$ (for the photospheric magnetic field).

The solar light from the $15 \mathrm{~cm}$ objective lens enters the polarimeter, which consists of two ferroelectric liquid crystals and a polarizer (we adopt the single-beam polarimetry), before folding mirrors. Polarized light enters into an Echelle spectrograph, and a spectrum is imaged on an infrared camera (XENICS XEVA-CL-640) with $640 \times 512$ pixels. The exposure time is set to $20 \mathrm{msec}$, and 96 polarization modulated images are taken at one slit position for two seconds. The integration of 96 images reduces the polarization noise to less than $10^{-3}$. The slit covers half of the solar diameter, and two east-west scans, are performed to cover the full-Sun. A full-Sun scan takes about two hours.

\section{Preliminary Results}

Here we show examples of the observations of polarization in filaments. A He I 10830 Stokes I map taken on 2011 Dec 10 is shown in Figure 1. Enlargements of major filaments (labeled 1-4 in the full-disk image) and linear polarization signals of the red component of the He I 10830 line, displayed with short lines showing the orientation and the degree of the polarization, are also shown in Figure 1. Linear polarizations in filaments are produced by atomic level imbalance under the presence of magnetic field (see e.g. Trujillo Bueno et al., 2002).

As seen in Figure 1, the polarization signals, namely the magnetic fields, are sheared. On the basis of the statistical study of the observed polarizations in filaments, the majority of the filaments are found to show the polarizations that the equator side shifts to 


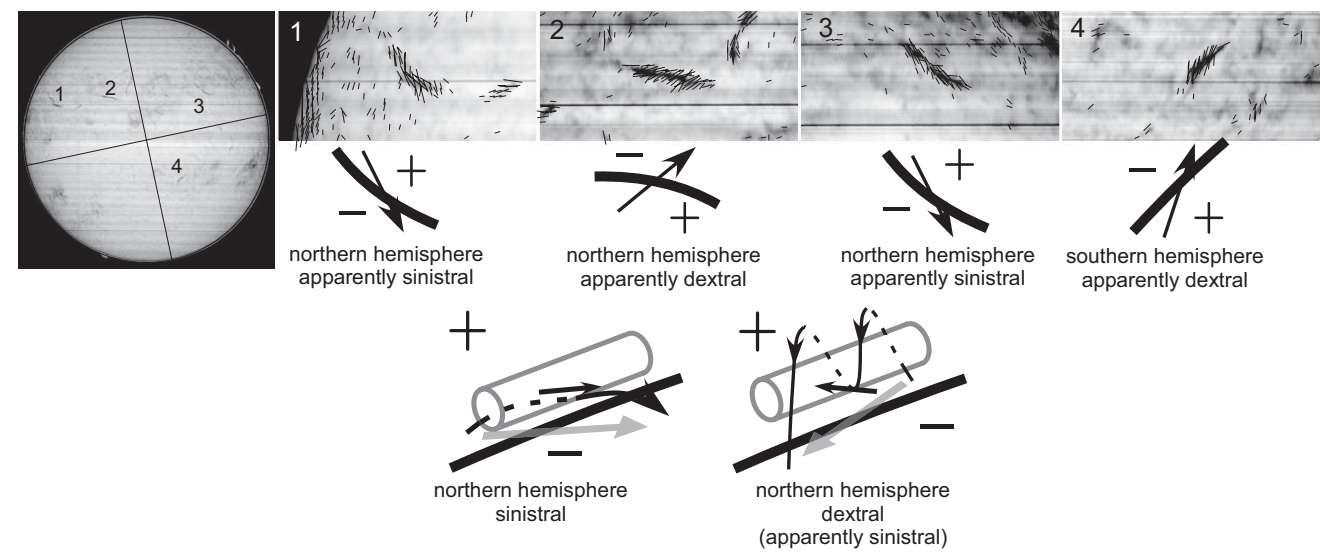

Figure 1. Top left: He I 10830 image on 2011 Dec 10. Major dark filaments are labeled 1-4, and their linear polarizations are shown in the top row with short black lines. Apparent magnetic connections presumed from the polarizations are shown below them. The cartoons in the bottom row show the possible interpretations of the observations. In the cartoons, black arrows show the magnetic field corresponding to the observed polarizations and gray arrows show the overall magnetic connection between the positive and negative magnetic regions.

the west (filaments 1, 3, and 4 show the polarization of the majority, and filament 2 is exceptional). This result seems to suggest that the majority of the filaments show sinistral (dextral) chirality in the northern (southern) hemisphere, as shown in the bottom-left cartoon in Figure 1. This is opposite to the interpretation by the former studies (Martin, 1998). However, if there is a flux rope with twisted magnetic field and filaments are located at the bottom of the flux rope as shown in the bottom-right cartoon in Figure 1, the apparent chirality of the magnetic field in the filament and the actual chirality of the overall magnetic connection are opposite to each other. In such a case, the overall magnetic connection corresponds to the dextral (sinistral) chirality in the northern (southern) hemisphere. Therefore, our result supports the view that filaments are supported by coronal cavity flux ropes (Berger, 2012).

This work was supported by a Grant-in-Aid for Scientific Research (No.17204014, 20052008, P.I.: T. Sakurai; and also partly by No.23244035, 2011-2014, P.I.: Y. Hanaoka) from the Ministry of Education, Culture, Sports, Science and Technology of Japan.

\section{References}

Berger, T. 2012, in T. Rimmele, A. Tritschler, F. Woger, et al. (eds.) The Second ATST-EAST Meeting: Magnetic Fields from the Photosphere to the Corona, ASP. Conf. Ser. 463 (San Francisco: ASP), p. 147

Hanaoka, Y., Sakurai, T., Shinoda, K., Noguchi, M., Miyashita, M., Fukuda, T., Suzuki, I., Hagino, M., Arai, T., Yamasaki, T., \& Takeyama, N. 2011, in J. R. Kuhn, D. M. Harrington, H. Lin, et al. (eds.) Solar Polarization 6, ASP. Conf. Ser. 437 (San Francisco: ASP), p. 371

Martin, S. F. 1998, in D. F. Webb, B. Schmieder, \& D. M. Rust (eds.) New Perspectives on Solar Prominences, ASP. Conf. Ser. 150 (San Francisco: ASP), p. 419

Sakurai, T., Ichimoto, K., Nishino, Y., Shinoda, K., Noguchi, M., Hiei, E., Li, T., He, F., Mao, W., Lu, H., Ai, G., Zhao, Z., Kawakami, S., \& Chae, J.-C. 1995, PASJ, 47, 81

Trujillo Bueno, J., Landi Degl'Innocenti, E., Collados, M., Merenda, L., \& Manso Sainz, R. 2002, Nature, 415, 403 